\title{
CONGENITAL DIAPHRAGMATIC HERNIA - A LATE PRESENTATION
}

\author{
Anjali Bharani, Hemant Jain. \\ Department of Pediatrics, Mahatma Gandhi Memorial Medical College, Indore, Madhya Pradesh, India.
}

\begin{abstract}
Congenital diaphragmatic hernia $(\mathrm{CDH})$ occurs due to incomplete muscularization of the diaphragm. While majority of these are diagnosed either antenatally or in the immediate new-born period in view of respiratory distress, a few may remain undiagnosed beyond neonatal period as they are asymptomatic or minimally symptomatic. We report two such cases who presented with respiratory distress and were diagnosed to have late presenting $\mathrm{CDH}$. An eight-year-old male child with weight of $12 \mathrm{~kg}$ presented with intermittent fever and cough for one-month, abdominal distension, and occasional bilious vomiting for 15 days and respiratory distress for 2 days. Ultrasonography of the chest revealed herniation of abdominal contents in the left chest cavity and sub-acute intestinal obstruction. He underwent surgery but succumbed to his illness. A 2-year-old female child came to the emergency department with fever, cough for 20 days and respiratory distress for 4 days. X-ray chest showed left large air-fluid filled cavity in the left lung. She underwent emergency laparotomy and a defect in the left hemi-diaphragm with herniation of stomach was noted. The hernia contents were reduced, and diaphragmatic defect closed. Post operatively she recovered well and was discharged after 7 days with full recovery.
\end{abstract}

\section{Introduction}

Congenital diaphragmatic hernia $(\mathrm{CDH})$ occurs in approximately 1 in every 2000 to 4000 newborns. 1 The most common defect is on left side (80-85\%) posterolaterally. ${ }^{2}$ More than $90 \%$ of these are either diagnosed antenatally or in the immediate new-born period in view of respiratory distress and are associated with significant mortality. ${ }^{3}$ However about a few cases may present beyond neonatal period. Late presenting $\mathrm{CDH}$ has been defined as $\mathrm{CDH}$ diagnosed after the neonatal period which may have been asymptomatic or only few symptoms in neonatal period and picked up on routine $X$-ray examination of chest beyond the neonatal period. ${ }^{2,4}$ The late presenting $\mathrm{CDH}$ is reported to have incidence of as high as $5-45.5 \%$ of all cases of $\mathrm{CDH} .{ }^{2,4}$ Late presenting $\mathrm{CDH}$ is relatively rare, often misdiagnosed, and hence gets delayed treatment. While the neonatal $\mathrm{CDH}$ carries high morbidity and mortality rates, the late-presenting $\mathrm{CDH}$ carries favourable prognosis if diagnosed early. We report case series of two cases presenting to our institute with this disorder and their outcome.

\section{Case 1}

An eight-year-old male child presented to the emergency room with intermittent fever and cough for one-month,

Address for Correspondence: Dr Anjali Bharani (Associate Professor) of Pediatrics, 119, Ravindra Nagar, Indore: 452018, Madhya Pradesh, India.

Email: dr.anjalibharani@gmail.com

(C)2021 Pediatric Oncall

\author{
ARTICLE HISTORY \\ Received 27 October 2020 \\ Accepted 29 December 2020
}

\section{KEYWORDS}

diaphragmatic hernia, late presentation abdominal distension, and occasional bilious vomiting for 15 days and respiratory distress for 2 days. He was unable to put on weight and had recurrent cough and cold but did not require any admission. Mother had tuberculosis (TB) 5 years ago for which she had received anti-tubercular treatment (ATT) for 6 months. On examination the child was malnourished with weight of $12 \mathrm{~kg}\left(<3^{\text {rd }}\right.$ percentile) and height of $100 \mathrm{~cm}\left(<3^{\text {rd }}\right.$ percentile). He was febrile and had significant distress with respiratory rate of $50 / \mathrm{min}$ and heart rate of $120 /$ min. There was no lymphadenopathy or edema, but oxygen saturation was $84 \%$ on room air and improved to $95 \%$ on oxygen by mask. On respiratory system examination, the air entry was markedly reduced on the left side and crepts were present on the right side. On cardiovascular examination, the apical impulse was felt in the right $4^{\text {th }}$ intercostal space and trachea was shifted to right side. On per abdomen examination, the bowel loops were dilated, and bowel sounds were absent. Chest X-ray PA and lateral view was done which showed a large air- fluid level cavity in left chest cavity and features of intestinal obstruction (Figure 1 and 2). Ultrasonography (USG) of the chest revealed herniation of abdominal contents in the left chest cavity and subacute intestinal obstruction. Hence suspecting a late presenting $\mathrm{CDH}$, child was taken up for emergency laparotomy. He was found to have multiple tubercular nodules on the intestines with lymphadenopathy and multiple adhesions and herniation of stomach in the left chest cavity was confirmed. Adhesiolysis was done and surgical reduction of left sided diaphragmatic hernia was done, and the defect closed. However, he succumbed to his illness on the next day of surgery. 
Figure 1 and 2. $X$ ray chest and abdomen PA and lateral view respectively showing large air-fluid filled cavity in left hemithorax with shift of mediastinum to the right side and features of intestinal obstruction

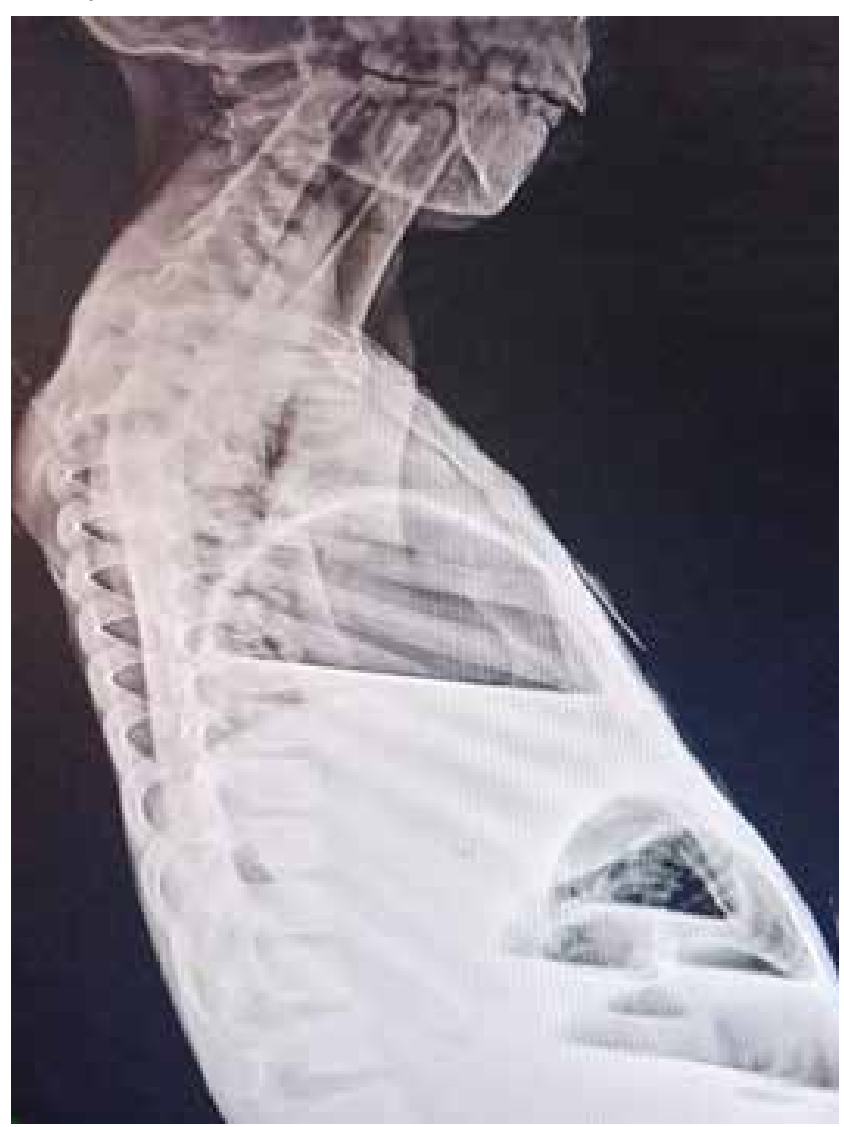

\section{Case 2}

A 2-year-old female child came to the emergency department with fever, cough for 20 days and respiratory distress for 4 days. She was asymptomatic before this episode. On examination she had significant respiratory distress with a heart rate: $130 / \mathrm{min}$ and respiratory rate of $50 / \mathrm{min}$. Her weight was $9 \mathrm{~kg}\left(<3^{\text {rd }}\right.$ centile). On respiratory system examination, air entry was reduced on the left side with shift of mediastinum to the right. Initial X-ray chest showed left large airfluid filled cavity for which chest tube was put. Repeat chest radiograph with nasogastric tube was done which showed tip of the naso-gastric tube in the left hemithorax clinching the diagnosis of $\mathrm{CDH}$ (Figure 3 ).

She underwent emergency laparotomy; herniation of stomach was noted through a defect in the left hemidiaphragm and reduction of same and closure of the defect was done. She was discharged after 7 days with full recovery.

\section{Discussion}

$\mathrm{CDH}$ results from incomplete closure of the normal pleuroperitoneal canal during the fetal development. ${ }^{1}$ They are classified based on the herniation time, the severity of pulmonary hypoplasia, survival, and the presence of late pulmonary sequelae. ${ }^{5}$ Type 1 refers to cases in which the herniation develops in the early period of rapid bronchial division. Hence it results in bilateral pulmonary hypoplasia and perinatal mortality. Type 2 refers to cases in which the

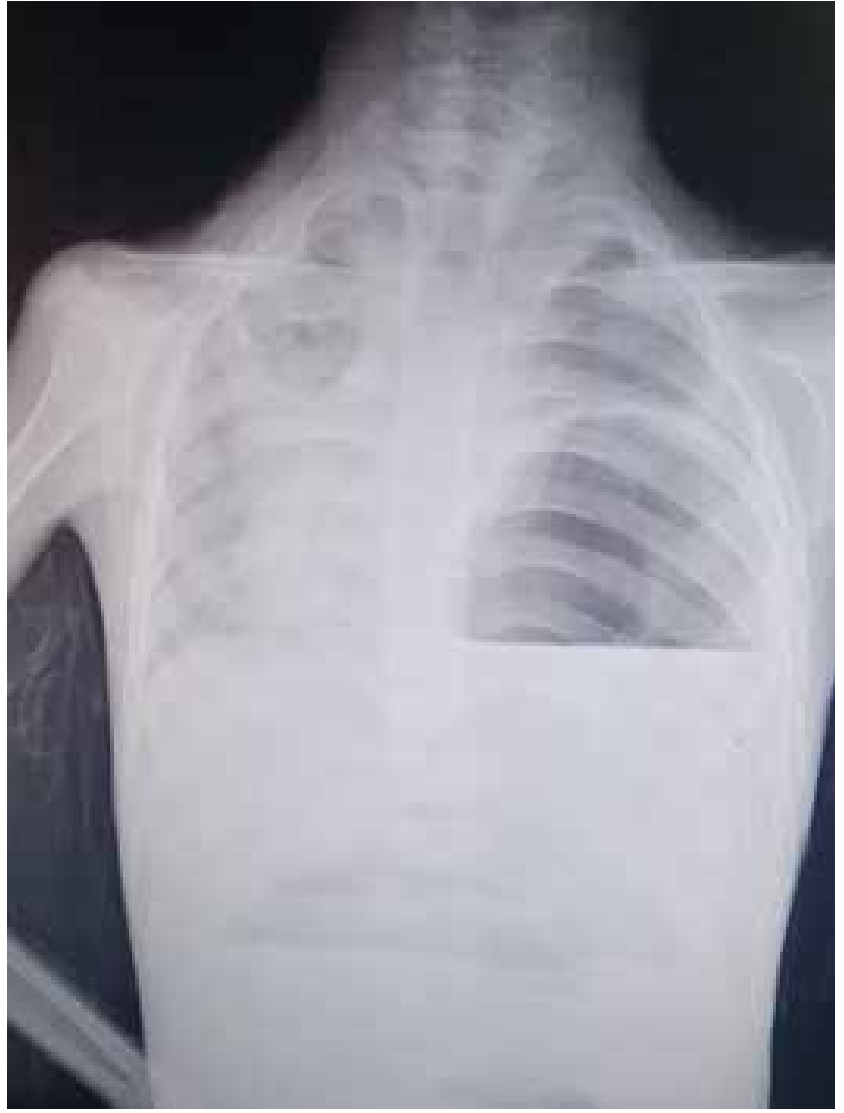

herniation develops in the late period of rapid bronchial division. Hence it results in unilateral pulmonary hypoplasia, and potential pulmonary hypertension. Type 3 refers to cases in which the herniation has occurred during either late gestation or early neonatal period. In them herniation might present at birth with mild respiratory distress. Pulmonary hypoplasia is minimal or absent and most of them survive with no detectable respiratory problems. In the type 4, the herniation occurs after the neonatal period and such patients have no pulmonary hypoplasia and survive with no respiratory problems. ${ }^{5}$ Especially in type 4 patients there may be no herniation until an episode of increased abdominal pressure causes herniation to develop. Both our patients had type 4 herniation as they had no pulmonary hypoplasia.

$\mathrm{CDH}$ may also be associated with other secondary abnormalities or congenital abnormalities. The secondary abnormalities commonly associated are malrotation of intestines and pulmonary hypoplasia. ${ }^{6}$ Cardiac abnormalities have been reported to be associated in such patients. ${ }^{6}$

Diagnosis is frequently made by chest $X$-ray, but it can mimic pneumothorax, cavity with air-fluid level, pleural effusion, or eventration of diaphragm, thereby leading to misdiagnosis. ${ }^{7,8,9,10}$, In such cases chest CT scan leads to confirmatory diagnosis and prevents delay in treatment.

As soon as the diagnosis is confirmed early surgical intervention should be sought to prevent unnecessary 
Figure 3. X-ray chest PA view of 2-year female child showing large air fluid level in left hemithorax with tip of Ryle's tube in the left chest suggestive of herniation of stomach. A mistakenly put chest tube can also be seen on left side

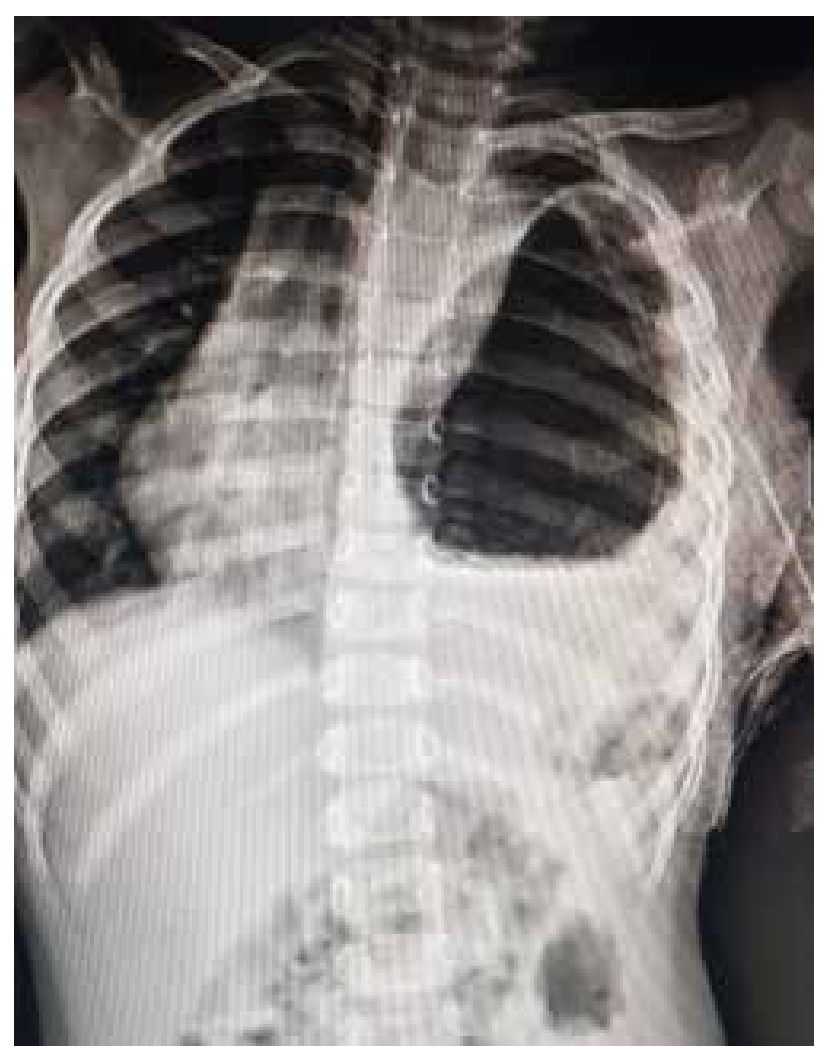

complications. Late presenting $\mathrm{CDH}$ has more favourable prognosis due to absence of pulmonary hypoplasia. ${ }^{6}$ Acute gastric volvulus associated with $\mathrm{CDH}$ is extremely rare but life-threatening complication. It is associated with elongation or absence of 2 of the 4 ligaments of stomach which are attached to the left diaphragm. Any increased abdominal pressure in such cases may lead to fatal consequences like gastric strangulation, ischaemia, perforation, pancreatitis, peritonitis, shock, and death with a mortality of $80 \% .{ }^{11,12}$ Late presenting $\mathrm{CDH}$ with gastric volvulus is often misdiagnosed as pneumonia with cavity formation, tension pneumothorax, empyema or pneumatocele. ${ }^{11,12}$ Unfortunately, this many times leads to iatrogenic error of inserting chest tube and causing life threatening complications. In our second patient, chest tube was inserted but it did not lead to any lifethreatening complications. Inappropriate tube insertion may lead to damage to intrathoracic abdominal viscera. There is also risk of spillage of gastric and abdominal contents into the mediastinum leading to mediastinitis or damage to blood vessels leading to haemorrhage. ${ }^{13}$ If $\mathrm{CDH}$ is suspected on plain radiograph, it can be easily diagnosed by placing a nasogastric tube and repeating the chest X-ray; in $\mathrm{CDH}$ the tip of the nasogastric tube can very well be seen in the thoracic cavity as was seen in our second patient. Whenever gastric volvulus is suspected surgery should not be delayed and early stomach decompression should be done to reduce the distension.

\section{Conclusion}

Late presenting $\mathrm{CDH}$ can have wide spectrum of clinical presentation and hence a high index of suspicion is required for diagnosis. Early pick up and treatment can prevent development of life-threatening complications like gastric volvulus. Early diagnosis and treatment can lead to good outcomes.

\section{Compliance with Ethical Standards}

Funding None

Conflict of Interest None

\section{References:}

1. Magnuson DK, Parry RL, Chwals WJ. In Selected Abdominal Gastrointestinal Anomalies. Editors: Martin RJ, Fanaroff AA, Walsh MC. Neonatal-Perinatal Medicine. Mosby Elsevier. Philadelphia, PA. 2006:1381-1417.

2. Baglaj M. Late presenting congenital diaphragmatic hernia in children. A clinical spectrum. Pediatr Surg Int. 2004;20:658-669.

3. Sridhar AV, Nichani S. Late presenting congenital diaphragmatic hernia. Emerg Med J. 2004;21:261.

4. Elhalaby EA, Abo Sikeena MH. Delayed presentation of congenital diaphragmatic hernia. Pediatr Surg Int. 2002; 18:480-485.

5. Wiseman NE, MacPherson RI. "Acquired" congenital diaphragmatic hernia. J Pediatr Surg. 1977; 12:657-665.

6. Kim DJ, Chung JH. Late-Presenting Congenital Diaphragmatic hernia in Children: The experience of Single Institution in Korea. Yonsei Med J. 2013;54: 1143-1148.

7. Baerg J, Kanthimathinathan V, Gollin G. Late presenting congenital diaphragmatic hernia: diagnostic pitfalls and outcome. Hernia. 2012;16:461-466.

8. Muzzafar S, Swischuk LE, Jadhav SP. Radiographic findings in late presenting congenital diaphragmatic hernia: helpful imaging findings. Pediatr Radiol. 2012;42:337-342.

9. Waseem M, Quee F. A wheezing child: breath sounds or bowel sounds? Pediatr Emerg Care. 2008;24:304-306.

10. Strunk T, Simmer K, Kikiros C, Patole S. Late-onset right sided diaphragmatic hernia in neonates-case report and review of literature. Eur J Pediatr. 2007;166:521-526.

11. Ayala JA, Naik-Mathuria B, Olutoye OO. Delayed presentation of congenital diaphragmatic hernia manifesting as combined-type acute gastric volvulus: a case report and review of literature. J Pediatr Surg. 2008;43:E35-39.

12. Vandy FC, Landrum JE, Gerig NR, Prahlow JA. Death Due to late-presenting congenital diaphragmatic hernia in a 2-year-old child. Am J Forensic Med Pathol. 2008;29:75-79.

13. Coren ME, Rosenthal M, Bush A. Congenital diaphragmatic hernia misdiagnosed as tension pneumothorax. Pediatr Pulmonol. 1997;24:119-121. 